\title{
Noise gratings in bleached silver halide diffuse-object holograms
}

\author{
A. Fimia and R. Fuentes \\ Laboratorio de Optica, Departamento Interuniversitario de Optica, Universidad de Alicante, Apdo. 99, Alicante E 03080, Spain
}

\author{
A. Beléndez
}

Departamento de Ingeniería de Sistemas y Comunicaciones, Universidad de Alicante, Apdo. 99, Alicante E 03080, Spain

Received March 14, 1994

\begin{abstract}
Noise gratings in diffuse-object holograms recorded as volume phase holograms in bleached silver halide emulsion are experimentally analyzed. Measurements of the diffraction efficiency and the noise of the holograms are taken. The experimental results obtained show that there is a drop not only in the diffraction efficiency but also in the noise that is due to the presence of noise gratings.
\end{abstract}

Bleached silver halide emulsions have long been used as a medium for recording volume phase holograms. Unfortunately, while the resulting phase hologram has a high diffraction efficiency, it is usually accompanied by an increase in the scattering of light and a consequent reduction in image quality. It is well known that the principal source of noise in phase diffuse-object holograms is the low-frequency random-phase pattern caused by the self-interference of light from the extended object ${ }^{1,2}$; however, in this Letter we show that noise gratings can be an important source of noise in diffuse-object holograms when the photochemical processing used introduces very small thickness changes.

Previous papers have reported the effects of different parameters on noise grating properties, such as the influence of the exposure and recording and readout wavelength $s^{3,4}$ on the efficiency of these gratings as well as the influence of polarization, ${ }^{5,6}$ beam ratio, ${ }^{7}$ photochemical processing, ${ }^{8-10}$ and coherence of the recording light. ${ }^{11}$ Furthermore, from a theoretical point of view, some models ${ }^{12,13}$ have been developed that have produced good agreement between experimental and theoretical results.

Nevertheless, all the aspects mentioned above regarding noise gratings have been studied with a single collimated beam or two collimated beams of light at recording, and with focusing elements such as holographic lenses, ${ }^{14}$ but to our knowledge the presence and the analysis of the characteristics of noise gratings in diffuse-object holograms have not been studied until now.

In this Letter we experimentally analyze noise gratings in diffuse-object holograms recorded in bleached silver halide emulsion. The aim of this study is to present experimental measurements of the diffraction efficiency and the noise and to show that noise gratings recorded during the formation of the hologram influence these holographic parameters.

The object used consisted of a uniformly transilluminated square glass diffuser $2 \mathrm{~cm} \times 2 \mathrm{~cm}$ with an opaque $1 \mathrm{~cm} \times 1 \mathrm{~cm}$ square in the center. The distance of the object from the recording medium was $30 \mathrm{~cm}$, and the reference beam was collimated to form a $37.5^{\circ}$ angle with the normal of the holographic plate, which was parallel to the object. The recording wavelength was $632.8 \mathrm{~nm}$ from a $\mathrm{He}-\mathrm{Ne}$ laser. Polarization was perpendicular to the plane of incidence, and the reference-to-object beam ratio was 5 . A series of holograms, for which the exposure ranged from 10 to $300 \mu \mathrm{J} / \mathrm{cm}^{2}$, was made with plates of Agfa Gevaert 8E75 HD, a fine-grain emulsion. We eliminated spurious reflections by placing an indexmatched absorbing layer against the glass side of the photographic plates. The exposed plates were developed in PAAAC developer. The developed plates were rinsed briefly and bleached without a fixation step. Two types of bleach bath were used in these experiments: R-10 and EDTA. Both of them are rehalogenating bleach baths. The resulting emulsion thickness change introduced with these rehalogenating baths is very small $(<0.05 \mu \mathrm{m})$ in the nominally 6- $\mu \mathrm{m}$-thick film. ${ }^{8}$ Also, it is assumed that the average refractive index does not change appreciably as a result of processing. ${ }^{8}$ Because the thickness and the average refractive index of the holographic recording material show little change when this chemical processing is done, the reconstruction geometry of the holograms corresponding to maximum diffraction efficiency will coincide with the construction geometry if the recording and the readout wavelengths are equal. This implies that Bragg's law will be complied with in the reconstruction stage. Details of the processing schedule as well as the developer and bleach formulas are given in Tables 1 and 2 .

Once the holograms were processed, the next step was to find their diffraction efficiencies and transmittances as a function of the reconstruction angle in order to show the presence of noise gratings. Processed holograms were replayed by the conjugate of the collimated reference wave, and the diffracted output beam formed the real image of the object. The noise was measured as the ratio between the light intensity in the central square of the reconstructed 
Table 1. Processing Schedule ${ }^{\alpha}$

Step Procedure

1. Develop

2. Rinse in running water

3. Bleached

4. Wash in running water

${ }^{a}$ All solutions at $20^{\circ} \mathrm{C}$.

Table 2. Developer and Bleach Bath Formulas

\begin{tabular}{lc}
\hline PAAAC developer & \\
Sodium carbonate & $120 \mathrm{~g}$ \\
Ascorbic acid & $18 \mathrm{~g}$ \\
Phenidon & $0.5 \mathrm{~g}$ \\
Distilled water to make & $1 \mathrm{~L}$ \\
R-10 Rehalogenating bleach bath & \\
Potassium dichromate & $2 \mathrm{~g}$ \\
Sulfuric acid & $10 \mathrm{~mL}$ \\
Potassium bromide & $35 \mathrm{~g}$ \\
Distilled water to make & $1 \mathrm{~L}$ \\
EDTA Rehalogenating bleach bath & \\
Ferric sulfate & $30 \mathrm{~g}$ \\
EDTA disodium salt & $30 \mathrm{~g}$ \\
Potassium bromide & $30 \mathrm{~g}$ \\
Sulfuric acid & $10 \mathrm{~mL}$ \\
Distilled water to make & $1 \mathrm{~L}$ \\
\hline
\end{tabular}

object and the incident light intensity. The diffraction efficiency, the transmittance, and the noise were measured as a function of the replay angle with the same wavelength and polarization used during the recording stage. Measurements were obtained at $1^{\circ}$ increments around the angle of the reference beam. In all cases we corrected the experimental measurements by taking into account the losses that were due to the reflection at the two surfaces of the plates. We expected the noise grating efficiency to be quite pronounced when no fixation step was used and that this would give rise to a reduction in the diffraction efficiency.

Plots of the diffraction efficiency and transmittance as functions of the reconstruction angle are shown in Fig. 1. Figure 1(a) corresponds to a diffuse-object hologram for which a fixation step was used in processing, whereas for Figs. 1(b) and 1(c) no fixation steps were used. As can be seen from Figs. 1(b) and 1 (c), peak diffraction efficiency is not observed at the
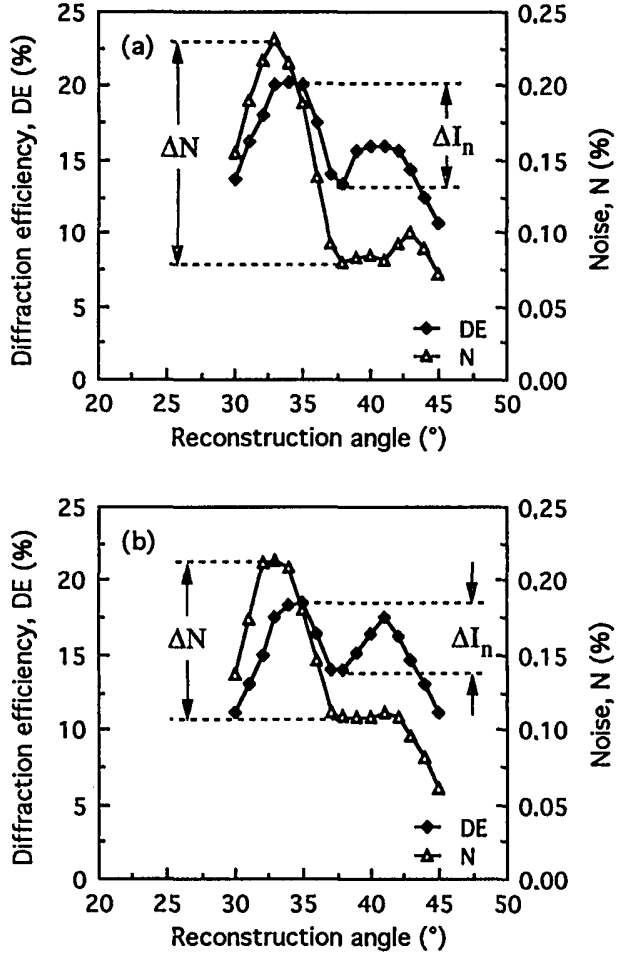

Fig. 2. Comparison of the diffraction efficiency and the noise for diffuse-object holograms processed without fixing and definition of the efficiency of the noise gratings $\Delta I_{n}$ and the influence of the noise gratings on the total noise $\Delta N$ : (a) when R-10 was used, (b) for the case of EDTA.

Bragg angle $\left(37.5^{\circ}\right)$, because there is drop in the efficiency for this reconstruction angle. This drop is due to the presence of simultaneously recorded scatter noise gratings, which were formed over the entire hologram surface by interference between the main recording beams and those scattered from the silver halide grains. In Fig. 1(b) the curve is not symmetrical around the recording angle and there is a little displacement in the angular response, which is not centered in the reference angle. Because the thickness and the average refractive index of the emulsion have not changed with the processing used, this displacement in the angular response could be caused by shear-type effects. ${ }^{14,15}$ However, in Fig. 1(a) the displacement in the angular response is due to the presence of considerable emulsion thickness.
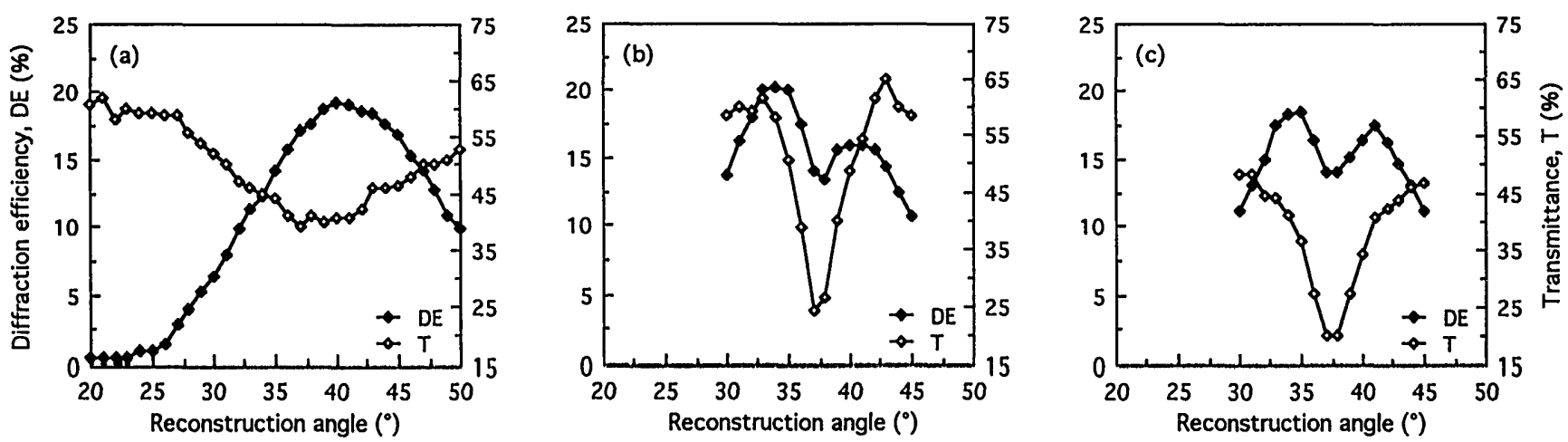

Fig. 1. Diffraction efficiency and transmittance as a function of the reconstruction angle: (a) when a fixation step was used and a level of exposure of $30 \mu \mathrm{J} / \mathrm{cm}^{2} ;$ (b), (c) when no fixation step was used and a level of exposure of $60 \mu \mathrm{J} / \mathrm{cm}^{2}$. 

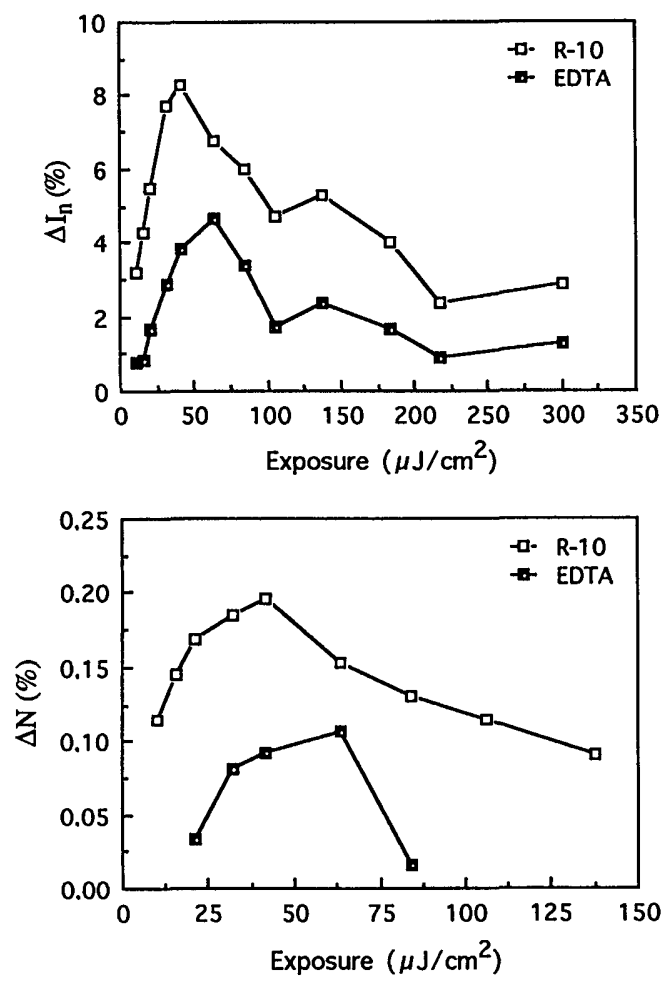

Fig. 3. Parameters $\Delta I_{n}$ and $\Delta N$ as a function of exposure.

In Fig. 2 we compare the diffraction efficiency and the noise for diffuse-object holograms processed without any fixing. As is shown in this figure, there is a drop that is due to the presence of noise gratings not only in the efficiency but also in the noise. Additionally, the curve for the noise is not symmetrical around the recording angle when an R-10 or EDTA bleach bath is used. To obtain a quantitative measurement of the intensity scattered by noise gratings we define the efficiency of the noise gratings, $\Delta I_{n}$, as the difference between the peak diffraction efficiency and the efficiency at the reference angle, as shown in Fig. 2. $\Delta I_{n}$ will be the maximum efficiency of noise gratings and corresponds to the reconstruction geometry in which Bragg's law is complied with for all elementary gratings of the noise gratings. A similar definition is considered for analysis of the drop in the noise due to noise gratings, $\Delta N$.

Figure 3 shows the calculated values for $\Delta I_{n}$ and $\Delta N$ as a function of exposure for replay at Bragg incidence. It can be observed that the loss of ef- ficiency that is due to noise gratings takes values between $2.36 \%$ and $8.20 \%$ when R-10 is used and between $0.78 \%$ and $4.64 \%$ in the case of EDTA. On the other hand, $\Delta N$ varies between $0.09 \%$ and $0.19 \%$ for R-10 and between $0.02 \%$ and $0.11 \%$ for EDTA. These data show that significant differences in $\Delta I_{n}$ and $\Delta N$ appear when the bleach bath is varied.

In summary, we have demonstrated the formation of noise gratings during the recording of diffuseobject holograms in bleached photographic emulsion. These noise gratings have a significant influence on the final characteristics of the diffuse-object holograms, especially as regards diffraction efficiency and noise. Finally, the experimental results of this Letter are important when holograms for optical storage or optical interconnections ${ }^{16}$ are recorded.

This research was supported by the Direcció General d'Ensenyaments Universitais i Investigació de la Generalitat Valenciana, Spain (project GV-1165/93).

\section{References}

1. J. Upatnieks and C. D. Leonard, J. Opt. Soc. Am. 60, 297 (1970).

2. A. Fimia, L. Carretero, and R. Fuentes, Appl. Opt. 31, 2408 (1992).

3. R. R. A. Syms and L. Solymar, Opt. Commun. 43, 107 (1982).

4. L. Wang and R. K. Kostuk, Opt. Lett. 14, 919 (1989).

5. R. K. Kostuk and G. T. Sincerbox, Appl. Opt. 27, 2993 (1988).

6. A. Beléndez, L. Carretero, and I. Pascual, Appl. Opt. 32, 7155 (1993).

7. L. Solymar and G. D. G. Riddy, J. Opt. Soc. Am. A 7, 2107 (1990).

8. R. K. Kostuk, Appl. Opt. 30, 1611 (1991).

9. A. Beléndez, I. Pascual, and A. Fimia, Opt. Quantum Electron. 25, 139 (1993).

10. L. Carretero, A. Beléndez, and A. Fimia, J. Mod. Opt. 40, 687 (1993).

11. A. Beléndez, L. Carretero, and A. Fimia, Opt. Commun. 98, 236 (1993).

12. G. D. G. Riddy and L. Solymar, Electron. Lett. 22, 872 (1986).

13. L. Carretero, A. Fimia, and A. Beléndez, J. Mod. Opt. 40, 1299 (1993).

14. A. Beléndez, R. Fuentes, and A. Fimia, J. Opt. (Paris) 24, 99 (1993).

15. A. Beléndez, I. Pascual, and A. Fimia, J. Opt. Soc. Am. A 9, 1214 (1992).

16. R. K. Kostuk, J. W. Goodman, and L. Hesselink, Appl. Opt. 26, 3947 (1987). 